Revue d'histoire de l'Amérique française

REVUE D.HISTOIRE DE L'AMÉRIQUE FRANÇAISE

\title{
La Communaulté des habitans de la Nouvelle France
}

\section{Marcel Delafosse}

Volume 5, numéro 1, juin 1951

URI : https://id.erudit.org/iderudit/801687ar

DOI : https://doi.org/10.7202/801687ar

Aller au sommaire du numéro

Éditeur(s)

Institut d'histoire de l'Amérique française

ISSN

0035-2357 (imprimé)

1492-1383 (numérique)

Découvrir la revue

Citer ce document

Delafosse, M. (1951). La Communaulté des habitans de la Nouvelle France.

Revue d'histoire de l'Amérique française, 5(1), 118-125.

https://doi.org/10.7202/801687ar d'utilisation que vous pouvez consulter en ligne.

https://apropos.erudit.org/fr/usagers/politique-dutilisation/ 


\section{DOCUMENTS INEDITS}

\section{LA COMMUNAULTE DES HABITANS DE LA NOUVELLE FRANCE}

Copies de pièces tirées des Minutes du Notaire Teuleron de La Rochelle, (Archives de la Charente Maritime, France) liasse de l'année 1651, où ces pièces avaient été déposées lors de l'établissement des titres d'emprunts, faits par la Communauté de Québec.

$I$ - Ordres pour le voyage de France, 1650.

II - Pouvoirs donnés au sieur Bourdon.

III - Ordres pour le voyage de France, 1651.

La pièce IV, antérieure et toute différente, est un contrat de vente de la terre Saint-Michel.

M. Dela Fosse, Archiviste de la Charente-Maritime, La Rochelle.

Minutes TEULERON, notaire à La Rochelle. Liasse de 1651.

Ordres pour le voyage de France 1650 de la part du Conseil estably par sa Majesté pour les affaires de la Nouvelle France.

Quiconque s'est chargé des affaires de la Communaulté aura ordre de n'en rien faire dans la poursuitte et détermination des affaires dans l'emprunt des deniers et quoy que ce soit d'importance sinon par l'advis ordre et direction du R.P. Hierosme Lallemant, lequel le Conseil a prié d'y avoir l'œil et auquel pour cet effect il a donné et donne plein et entier pouvoir.

Il aura l'œil à faire la visitte en France des castors quy auront esté embarqués sans ordre du magasin lequel il fauldra confisquer, Monsieur Rozee pouroit faire arester pour cet effect tous les castors affin que ayant vérifié ce quy aura esté donné par le magasin à ung chacun le surplus soit jugé confisquable. 
Sur les trois blanes signés quy auront esté envoyés de la part du Conseil il fauldra faire choix d'une personne à Rouen comme Messieurs Rozée Guenet ou aultres pour faire la vente des castors envoyés pour le compte des créanciers faire leurs payements et si il y reste quelque chose il fauldra le faire tenir à La Rochelle et acquitter les billets quy y sont demeurés. On a escrit à Monsieur Rozée qu'on le priroit d'avoir l'œeil à cette vente et à ces payements.

Il fault aussy faire choix d'un homme à La Rochelle luy envoyer l'extrait des comptes du sieur Godefroy et ce qui concernera le sieur Pepin pour compter avec luy et luy faire payement sy on peult luy faire tomber [?] de l'argent ou de ce quy luy sera deub en faire promesse.

Estant arrivé au Havre le meilleur sera d'aller promptement à Rouen tant pour faire le choix cy-dessus que pour induire le sieur Rozée ou autres à faire l'acquit des billets restants en France de l'an passé.

Voir aussi le sieur Rozée si il vouldra accepter les conditions quy luy ont esté envoyés pour l'embarquement prochain, si il en fait refus il fauldra s'adresser à d'aultres soit en Normandie soit à La Rochelle.

Messieurs Rozée, Guenet ou autres quy se chargeront de l'embarquement de l'an prochain pour fournir au magasin le contenu dans le memoire des choses quy sont nécessaires audit magasin et quy advanceront l'argent quy sera nécessaire pour le payement des rescriptions de l'année 1649 et de l'année 1650 et pour l'acquit des autres debtes de la communaulté pouront estre assurés dans la convention qu'on fera avec eux ou dans l'obligation qu'on leur en passeroit que les castors quy seront au pouvoir de la communaulté leur seront réservés privativement à tous autres navires quy pourroient venir à Québec en sorte qu'on ne fera aucune diversion des castors sinon dans l'asseurance qu'on aurait d'avoir de quoy fournir aux dits sieurs Rozée ou autres quy auroient advancés leurs deniers pour l'accomplissement du susdit mémoire et pour le paiement des debtes de la communaulté ou pour quelques autres emprunts que ce soit qu'il sera nécessaire de faire pour les besoins de ladite communaulté, c'est pourquoi on fera le possible pour donner advis par les premiers navires de tout ce quy auroit esté faict et accordé en ce point.

Si oultre les ordres du susdit R.P. Hierosme Lallemant le député du Conseil faisoit aulcun emprunt de deniers il scaura qu'il en demeurera responsable en son propre et privé nom comme aussi de quelques sortes d'affaires que ce soit qu'il auroit gérés contre les ordres dudit R.P. Hierosme Lallemant.

Il ne consentira point au payement des créanciers que leur procureur ne promette leur rendre les obligations et grosses adventures quy sont entre les mains du sieur Tallemant, le plus promptement que se pourra faire le voiage de Paris sera le meilleur affin d'avoir le consentement et approbation de Messieurs les commissaires de remplir la procuration du nom de celuy qui sera jugé à propos pour pouvoir agir et s'opposer à ceux qui vouldroient agir contre la communaulté, compter et arrester avec tous ceulx 
avec lesquels on est obligé et enfin pour faire en sorte que pour les affaires passées il n'y ait plus rien à demesler en France et que de tout on aye.l'esclaircissement nécessaire, qu'on retire toutes fois les obligations arrests de conseil billets, comptes et autres papiers quy doibvent estre mis au greffe du conseil de Québec.

Il fault compter avec le sieur Chaugeon d'une recognoissance rapportée par Monsieur Godefroy.

Il fault savoir quel compte a rendu le sieur de Chavigny de sept mil livres qu'il a recus dont Monsieur Godefroy s'est chargé de 1.8001.

Il fault prendre chez le sieur Teuleron la carquaison du Navire neuf et les radoubs qu'a fait le sieur de La Ferté.

Donnera avis à celuy quy aura charge de compter avec le sieur Pepin à La Rochelle qu'il doibt rejeter les parties de la somme de trois cents livres qu'il dict avoir payés au sieur Stevenot puisque tous les fraiz de créanciers sont payés dans la transaction.

Item les partyes de cent vingt cinq livres qu'il dict avoir payés à Paris pour raisons cy dessus.

Item la partye de deux cent livres pour les fraits pour avoir esté compris dans laditte transaction.

Il sera bon d'apporter en argent monnoyé pour le magasin la somme de huit à dix milles livres qu'on prendra aux conditions les moins onéreuse qu'il sera possible.

Sy on peult fortifier le païs du moins de 40 à 50 soldats quy seroient ung second camp volant pour la deffence du païs, c'est une chose souhaitable.

Il aura soing de faire venir dix ou vingt soldats pour le camp volant de 40 soldats quy est desja sur le païs affin de remplacer ung pareil nombre de soldats quy seroient renvoyés dudit camp volant aprés l'arrivée des navires mais que ce soient soldats bien choisis et hommes effectifs.

Il aura soing de faire venir des mathelots quy seront de service dans les navires quy viendront et quy seront loués à ung prix raisonable pour demeurer icy sur le païs et desquels les places seront remplacés par les mathelots quy sont maintenant icy et quy seront renvoyés en France.

Les présents ordres ont esté faicts et arrestés au Conseil à Québec le vingt neufviesme octobre 1650 - Par le Conseil, Audouart secrétaire.

Minutes Teuleron, notaire à La Rochelle. Liasse de 1651

\section{Pouvoirs donnés au sieur Bourdon}

Nous Jean de Lauson conseiller ordinaire du Roy en ses conseils d'Estat et privé et direction de ses finances, gouverneur et lieutenant général pour le Roy de la Nouvelle France et Hierosme Lallemant supérieur de la mission de la compagnie de Jésus audit païs de la Nouvelle France, sur la remonstrance qui nous a esté faicte par le sieur Jean Bourdon habitant dudit paīs que par la procuration generalle à luy donnée par Messieurs du conseil 
estably par le Roy pour les affaires de la communaulté des habitans dudit paìs à Québec en datte du vingt neuf octobre dénnier signé Audouart secrétaire dudit conseil il n'est pas dit en termes expretz qu'il pourra emprunter deniers à grosse adventure sur les marohandises qu'il convient charger la présente année dans ung ou plusieurs navires pour envoyer audit païs de la Nouvelle France, cela n'ayant pas été prévu par le conseil dudit Québec, neantmoins étant necessaire de donner toute asseurance à ceux qui feront tout ou partie dudit prest, adjoustans au pouvoir dudit sieur Bourdon nous avons authorisé et authorisons par ces présentes ledit sieur Bourdon et luy donnons plein pouvoir d'emprunter à la grosse adventure les sommes de deniers qui luy seront nécessaires pour le payement des marchandises qu'il lui convient charger pour ladite communaulté dans les navires nommés la Vierge et le Saint Joseph frettés par notre ordre et ce à telz profitz qu'il en pourra trouver, passer obligations et obliger par icelle les biens de ladite communaulté des habitans de la Nouvelle France speciellement lesdites marchandises ensembles les pelleteries qui seront chargées pour le retour, sans que la géneralité préjudicie à la spécialité ny au contraire, promettant avoir pour agréable ce qui sera geré et negocié par ledit sieur Bourdon, mesme de faire rendre et payer les sommes qui seront par lui empruntées en vertu de la susdite procuration et des présentes avecq les profits de la grosse adventure, en foy de quoy nous avons signé ces présentes et icelles faict signer à nos requestes à Pierre Teuleron notaire royal en ceste ville de La Rochelle le vingt sixiesme jour de may mil six cens cinquante ung.

(signé): De Lauson

Hierosme Lallemant

Minutes Teuleron, notaire à La Rochelle. Liasse de 1651

Extrait des registres du Conseil estably à Québec

par sa Majesté

[ 1651 ]

Instruction au sieur Jean Garos marchand de La Rochelle quy est prié de prendre la commission des affaires de la communaulté des habitans de la Nouvelle France.

Ledict sieur Garos recepvera des mains des capitaines Boucher et Boisleau les pelleteries envoyés de Québec suyvant les connaissements desdictz capitaines puis il mettera ez mains de mesieurs Pagetz et Beraudin lesdictz castors suivant la vante quy leur en a esté faitte et au poidz convenu par le marché fait entre eux à La Rochelle.

Et d'aultant qu'encores que nous ayons retenu les navires jusques à ce jour quinziesme de novembre quy est une saison toute extraordinaire les castors de Montréal ne sont pas arrivés et que d'ailleurs nous craignons que la rivière se trouvant barrée de glaces les navires les hommes et les castors embarqués n'aillent en perdiction nous envoyons ce que nous avons quy se 
monte a treize mil cent trentes et une livre pesant de castors, bien maris de n'en pouvoir envoyer davantage.

Pour réparer en quelque fasson ce deffault nous donnons pouvoir audit sieur Carrou [ sic ] d'engager et vendre auxdits sieurs Pagetz et Beraudin tous les castors de la communaulté de l'an prochain 1652 aux conditions cy-aprés expliqués excepté la rente seigneurialle de mille livres de castors deubs à messieurs de la Compaignie de la Nouvelle France et les trois milles livres de castors cy devant vendus auxdits sieurs Pagetz et Beraudin lesquels Dieu aydant leur seront fournis et livrés en leur temps suivant le marché.

Ainsy nous promettons de bonne foy à Messieurs Pagetz et Beraudin de faire tout notre possible à ce qu'il ne se fasse aucun divertissement de castors à leur prejudice et qu'il n'en soit donné à aucun navire quel qu'il soit

Nostre intention estant de paier les debtes du païs au plus tost et qu'il s'aquitte envers les créanciers nous désirons seulement que sur les castors de l'an prochain mil six cens cinquante et deux Messieurs Pagetz et Beraudin nous envoyent en danrées jusques à la fourniture de deux petites factures cy jointes signees de Lauson et Audouart segretaire du Conseil lesquelles denrées seront payées en castors ainsy qu'il sera dict cy après et que le surplus desdicts castors soit livré l'an prochain et ensuitte retenu par lesdictz sieurs Pagetz et Beraudin pour le prix d'iceux estre employé à l'acquist desdicts debtes.

Seront les dictes denrées et marchandises livrés aux risques desdicts sieurs Pagestz et Beraudin sur le quay de Québec bien conditionnés à tant pour cent du prix coustant et ses marchandises seront par nous payez ycy en castors au prix quallité et condiction de ceux de l'an 1651 à la reserve que nous nous contentons de vendre huict livres cinq soltz le castor moyttie gras et moyttié maigre et le reste des autres castors à proportion de leur quallité selon qu'il est spécifié au marché fait pour l'année 1651 et lesdicts castors yront en France aux risques desdicts sieurs Pagestz et Beraudin en considération de la diminution que nous faisons du prix des castors de l'an 1651.

Quant est de tant pour cent Messieurs Pagestz et Beraudin se souviendront qu'ils nous demanderent quattre vingtz pour cent et que nous offrimes soixante et dix, nous les prions de se contenter de soixante et quinze livres pour cent de toutes les marchandises comprises aux susdictes deux factures et le surplus des castors restant de l'année 1652 sera vendu à ses messieurs au mesme prix et condition que ceux de la presente année 1651 par ledict sieur Garroz auquel nous donnons pouvoir sy mieux n'ayment lesdicts sieurs Pagestz et Beraudin les prendre à leurs risques sur le quay de Québec sur le pied de huict livres cinq sols pour castor moytié gras et moittié maigre et le reste à proportion comme dessus.

Ledict sieur Garoz des deniers provenantz de la vante des castors de ceste année 1651 en payra le fret à ceux ausquels il est deub et les debtes privilégiées. 
Et d'aultant que nous désirons recognoistre par touttes voys l'assistance que messieurs Pagestz et Beraudin ont rendue à la communaulté sur ce que le sieur Bourdon nous a fait entendre qu'outre la fourniture du magazin ses messieurs lưy auront proposé de s'associer avecq luy à faire venir des marchandises à leur compte particulier pour estre lesdictes marchandises et danrees vandus ycy aux habitans mesme payables en castors, le Conseil donne pouvoir ausdicts sieurs Pagestz et Beraudin de faire venir ycy tel navires qu'ils jugeront a propos outre celuy quy aportera les denrées du magasin mentionnés dans nos factures et est permis au sieur Bourdon de s'y associer sy lesdictz sieurs Pagestz et Beraudin le jugent ainsy pour leur advantage.

Lesdictz sieurs Pagestz et Beraudin sont priés de faire prest à la communaulté à la grosse adventure ou autrement de la somme de neuf mille livres pour payer quelques rescriptions suyvant l'estat que nous envoyrons signe de Monsieur le Gouverneur et du segretaire du Conseil.

Et comme nous faison touts effortz pour acquitter la communaulté de la Nouvelle France ceste année quelques partyes en prenant sur eux et sur leur credict la fourniture de leurs necessités et provisions et faisant tenir à leurs fraix par quelques navires ce quy leur est necessaire au soulagement de la communaulté lesdictz sieurs Pagestz et Beraudin sauront que le paiement de ses denrées se fera en argent et non en castors.

Comme aussy ledistz sieurs Pagestz et Beraudin seront advertis que l'on se dispose de faire venir des hommes de travail de France pour le secour du pais et d'autant que les Normands ne boivent point de vin et sont d'un paīs plus froid que le Poictou le Conseil a pris résolution de les faire venir de Normandie mais encore lesdits sieurs Pagestz et Beraudin seront advertis que ceste despance se payra en argent et non en castors, nostre instruction estant que toute la pelleterie de l'an prochain sans aucun divertissement tombe es mains desdicts sieurs Pagestz et Beraudin à quoy nous travaillerons de bonne foy et Dieu aydant la communaulté sortira avecq honneur de toutes les debtes ausquelles elle est obligée ausquelles elle a engagés ses amis. Faict au Conseil tenu à Québec ce quinziesme jour de novembre mil six cent cinquante et ung.
(signé): de Lauson
Audouart, secrétaire.

Minutes de Teuleron notaire à La Rochelle Registre $1647, \mathrm{f}^{\circ} 44$

1647, 21 Mars.

Transport de Puisseaux Juchereaux [ de la terre de Saint Michel au Canada]

Sachent tous que par devant Pierre Teuleron notaire tabellion royal et garde notte héréditaire en la ville et gouvernement de La Rochelle ont esté presens en leurs personnes Pierre de Puiseau escuyer sieur de Sainte 
Foy demeurant de present en ceste ville d'une part, et honorable homme Noel Juchereau sieur des Chastellets faisant tant pour luy que pour honorable homme Jean Juchereau l'aisné sieur de More son frere comme ayant charge de luy ainsi qu'il a déclaré d'autre part, entre lesquelles partyes a esté fait et passé le contrat de vente cession et transport qui s'ensuit, c'est assavoir que ledit sieur de Puyseau de son bon gre et volonté a pour luy et les siens vendu ceddé et transporté à perpetuité savoir est le lieu et terre de Saint Michel se consistant en maisons grange et ung corps de logis qui avoit acoustumé de servir de brasserie et de forge, une chapelle, courts, jardins, terres labourables et non labourables, bois de haulte fustaye avec toutes leurs apartenances et dependences quelconques et autant qu'il a droit d'en jouir et posseder en consequence de la concession à luy faicte par Messieurs de la Grande Compagnie de la Nouvelle France en ce qui concerne ledit lieu de Saint Michel sans aulcune chose se retenir ny reserver, confrontant d'une part au grand fleuve Saint Laurent, d'autre part à la grande allée ou route, d'une autre part à la terre de Sillery apartenant aux Reverends Peres Jésuites de la Compagnie de Jésus et d'autres aux terres non encore concédées appartenant à ladite Compagnie à la charge de payer et continuer par lesdits sieurs Juchereau les cens et debvoirs deubs à ladite Compagnie et mentionnés par ladite concession datée du sept février mil six cens trente sept signée Lamy secretaire de ladite Compagnie dont et duquel dit lieu et terre de Saint Michel et de tout droit de propriété qui audit sieur de Puiseau peut composer et apartenir tant en conséquence de ladite ccncessicn que tout autrement il s'en est pour luy et ses successeurs desmis devestu et desaisi et a saisi et vestu lesdits sieurs Juchereau et iceux mis en bonne possession et saisine reelle et actuelle pour par eux et les leurs en jouir à leur volonté et à perpetuité, ladite vente et transport faite pour et moyennant la somme de six mille cinq cens livres tournois, de laquelle ledit sieur Juchereau esdits noms en a payé présentement audit sieur de Puiseau la somme de trois mille cinq cens livres tournois en pistolles escus d'or et monoye du cours de l'ordonnance comptée et nombrée à la veue dudit notaire et des tesmoins bas nommés et le restant qui est trois mille livres ledit sieur Juchereau esdits noms et solidairement renonçant aux bénéfices de division d'ordre de droit eviction et de discution de biens promet en faire payement audit sieur de Puiseau ou à son ordre dans XXX d'huy en six mois prochains pour tout delay avecq l'interest d'icelle à la raison du denier vingt, moyennant quoy promet et s'oblige ledit sieur de Puiseau de garantir et deffendre les dits lieux cy dessus vendus et transportés de bon et loyal gariment... [ suivant les formules habituelles ]. Faict et passé à La Rochelle en la maison du sieur Guillaume Feniou marchand d'icelle avant midi le vingt uniesme jour de mars mil six cens quarante sept, presents les sieurs Jean et Nicolas Juchereau fils du dit sieur de More, les sieurs Feniou.

[ signé ]: Pierre de Puiseau - Juchereau - Juchereau saint Denis - Juchereau La Ferté - Feniou - F. Pichon G. Feniou - Teuleron, notaire royal. 
[ en marge ]: Aujourd'huy ledit sieur de Puyseau desnommé au contrat de transport dont ces presentes sont en marge en sa personne a receu comptant dudit sieur Juchereau aussi y denommé present et stipulant la somme de trois mille livres tournois en principale d'une part restant de six mille cinq cens livres pour le prix de la terre de Saint Michel et cent douze livres dix sols d'autre part pour les interests des trois mille livres restantes, desquelles deux sommes revenant en une à celle de trois mille cent douze livres dix sols ledit sieur de Puyseau s'est contenté et en a quitté ledit sieur Juchereau et tous autres par cette quittance qu'il a signée et fait signer à sa requeste audit notaire royal soubsigné à La Rochelle étude dudit notaire apres midy le vingt neuviesme jour de décembre mil six cens quarante sept presents Jean Bortuste et Gilles Barre clercqs demeurant en icelle

$$
\text { (signe): Pierre de Puyseau - Barre - Bortuste - Teuleron. }
$$

Histoire du Canada français (cours d'histoire professé à la radio par le Chanoine Lionel Groulx) tome 1er, in-12, 222 pages. En vente dans toutes les librairies, en particulier, à la librairie de l'Action Nationale, 422 est, Notre-Dame, Montréal. Prix : édition régulière, $\$ 2.00$ (presque épuisée). Edition de luxe, chaque exemplaire autographié par l'auteur: \$3.50. Le deuxième tome paraîtra en septembre 1951 ; les 3e et $4 e$ en 1952. 